\title{
Microbiology Susceptibility Original Result
}

National Cancer Institute

\section{Source}

National Cancer Institute. Microbiology Susceptibility Original Result. NCI Thesaurus.

Code C87935.

The value in which the outcome of the microbiology susceptibility assessment observation was originally reported. 